\title{
The Gut Microbiome and Hepatocellular Carcinoma: Implications for Early Diagnostic Biomarkers and Novel Therapies
}

\author{
Yongbo Kang ${ }^{a, b}$ Yue Cai ${ }^{a}$ Ying Yang ${ }^{a}$ \\ aDepartment of Endocrinology, Affiliated Hospital of Yunnan University, Kunming, China; \\ bDepartment of Microbiology and Immunology, School of Basic Medical Sciences, Shanxi Medical \\ University, Taiyuan, China
}

\section{Keywords}

Hepatocellular carcinoma - Gut microbiota .

Microbiome-gut-liver axis · Diagnostic biomarker .

Potential therapeutic strategies

\begin{abstract}
Hepatocellular carcinoma (HCC) ranks the third place among all causes inducing cancer-associated mortality, worldwide. HCC nearly exclusively occurs in cases suffering from chronic liver disease (CLD), which results from the vicious cycle of liver damage, inflammation, and regeneration possibly lasting for dozens of years. Recently, more and more investigation on microbiome-gut-liver axis enhances our understanding toward how gut microbiota promotes liver disease and even HCC development. In this review, we summarize the mechanisms underlying the effect of gut microbiota on promoting HCC occurrence, with the focus on key pathways such as bacterial dysbiosis, leaky gut, bacterial metabolites, and microorganism-related molecular patterns, which promote liver inflammation, genotoxicity, and fibrosis that finally lead to cancer occurrence. Furthermore, we discuss gut microbiota's important potential to be the early diagnostic biomarker for HCC. Gut microbiota may be the candidate targets to simultaneously prevent CLD and
\end{abstract}

karger@karger.com www.karger.com/lic

Karger $\stackrel{\text { ! }}{=}$

GOPEN ACCESS
C 2021 The Author(s).

Published by S. Karger AG, Basel

This is an Open Access article licensed under the Creative Commons Attribution-NonCommercial-4.0 International License (CC BY-NC) (http://www.karger.com/Services/OpenAccessLicense), applicable to the online version of the article only. Usage and distribution for commercial purposes requires written permission.
HCC occurrence among advanced liver disease cases. We outlook the gut microbiota-targeting treatments in detail to prevent CLD and HCC progression.

(c) 2021 The Author(s).

Published by S. Karger AG, Basel

\section{Introduction}

Liver cancer (LC) is one of the most common malignant tumors in the world. In 2018, there were 841,080 new cases and 781,631 deaths $[1,2]$. With regard to the morbidity and mortality, LC ranks the seventh and third places across diverse cancers, which has caused a great cancer burden, globally $[1,3,4]$. Hepatocellular carcinoma (HCC) represents a frequently occurring primary LC [5]. It is estimated that the HCC morbidity will show an increasing trend by the following 10 years [6]. Generally, HCC is induced by chronic liver disease (CLD) [7], and its major risk factors include hepatitis $\mathrm{B}$ or $\mathrm{C}$ virus infection, diabetes, nonalcoholic fatty liver disease (NAFLD), alcoholism, as well as additional genetic or metabolic disorders $[8,9]$. HCC has a complicated pathogenic mecha-

Yongbo Kang and Yue Cai contributed equally.
Correspondence to:

Yongbo Kang, 657151276@qq.com

Ying Yang, yangying2072@126.com 
Table 1. Changes in microbiota composition associated with HCC in animal models

\begin{tabular}{|c|c|c|c|}
\hline Mice & DEN induced HCC & Altered gut microbiota & [23] \\
\hline Mice & STZ-HFD induced NASH-HCC & $\begin{array}{l}\text { Atopobium spp. } \uparrow, \text { Bacteroides spp. } \uparrow, \text { Bacteroides vulgatus } \uparrow, \text { B. acidifaciens } \uparrow, \text { B. uniformis } \uparrow \text {, } \\
\text { Clostridium cocleatum } \uparrow, \text { C. xylanolyticum } \uparrow, \text { Desulfovibrio spp. } \uparrow\end{array}$ & {$[25]$} \\
\hline Mice & HFHC induced NAFLD-HCC & $\begin{array}{l}\text { Mucispirillum } \uparrow \text {, Desulfovibrio } \uparrow \text {, Anaerotruncus } \uparrow \text {, Desulfovibrionaceae } \uparrow \text {, Bifidobacterium } \downarrow \text {, } \\
\text { Bacteroides } \downarrow\end{array}$ & {$[27]$} \\
\hline Mice & DMBA-HFD induced HCC & Altered gut microbiota & [28] \\
\hline Mice & MYC transgenic spontaneous HCC & $\begin{array}{l}\text { Gram-positive bacteria } \uparrow \text {, Bacteria mediating primary-to-secondary bile acid conversion } \uparrow \text {, } \\
\text { Clostridium scindens } \uparrow\end{array}$ & {$[32]$} \\
\hline
\end{tabular}

HFHC, high-fat/high-cholesterol.

nism; the etiology and exact molecular mechanism of HCC has yet to be fully elucidated to date.

As revealed by studies published over the last 10 years, gut microbiota has an important role in human health. Typically, gut microbiota substantially benefits its host, especially in terms of immunity and metabolism $[10,11]$, but gut microbiota has been increasingly recognized to be related to disease processes [12]. Recently, investigation on the microbiome-gut-liver axis enhances our understanding toward gut microbiota's effect on promoting liver disease occurrence and development, with bidirectional communication between the liver and gut microbiota. Gut microbiota together with their metabolites and products are suggested to have key functions in HCC occurrence. Meanwhile, emerging evidence suggests that the intestinal commensal bacterial species may exert the role of pathogenesis or protection during HCC development. Although still a relatively new area of research, the existing studies indicate that gut microbiome may be the candidate target used to prevent and manage HCC.

In addition, it is of critical importance to diagnose HCC cases at the early stage, so as to improve the patient prognosis [13]. However, HCC is usually diagnosed at the late stage that is generally accompanied with hepatic insufficiency and failure [5]. Gut microbiota is advantageous in diagnosing disease, including its noninvasive nature, great efficiency, and accuracy. Furthermore, more and more studies suggest that intestinal microbiota can serve as the biomarker to diagnose numerous disorders, including type 2 diabetes mellitus, pancreatic cancer, colorectal cancer, heart failure, liver cirrhosis (LC), as well as other central nervous system diseases [14-19]. Thus, gut microbiota may be potentially used to diagnose HCC at the early stage.

The present review first summarizes the existing studies that analyzed intestinal microbiota's possible function in HCC occurrence and progression. Then, we discuss gut microbiota-mediated treatment and early diagnosis for HCC. Some possible highlights for diagnosis and treatment, which could be used for future clinical applications, are included.

\section{Function of Gut Microbiota in HCC}

HCC is usually the result of a chronic disease process of the liver and almost never occurs spontaneously in the absence of liver disease. In addition, about $80-90 \%$ of HCCs occur in advanced fibrotic or cirrhotic livers, which means that about one-third of patients with compensated LC develop HCC in their lifetime [20, 21]. The hepatitis $\mathrm{B}$ or $\mathrm{C}$ virus-induced cirrhosis stands for a main risk factor leading to HCC. Nonetheless, with the increased morbidity rates of NAFLD and obesity among the developed countries, HCC has become more and more common in such areas [22]. Chronic liver inflammation is associated with HCC occurrence in patients with viral hepatitis, but the precise mechanism underlying HCC occurrence among NAFLD cases remains unclear. 
Table 2. Changes in microbiota composition associated with HCC in human studies

\begin{tabular}{|c|c|c|c|}
\hline Models & Disease & Implicated microbiota & Reference \\
\hline Human & $\mathrm{HCC}$ & Escherichia coli $\uparrow$ & [34] \\
\hline Human & $\mathrm{HCC}$ & $\mathrm{D}_{\text {dys }} \uparrow$, Proteobacteria $\uparrow$, Desulfococcus $\uparrow$, Enterobacter $\uparrow$, Prevotella $\uparrow$, Veillonella $\uparrow$, Cetobacterium $\downarrow$ & {$[35]$} \\
\hline Human & PLC & $\begin{array}{l}\text { Enterobacter ludwigii } \uparrow, \text { Enterococcaceae } \uparrow, \text { Lactobacillales } \uparrow, \text { Bacilli } \uparrow, \text { Gammaproteobacteria } \uparrow \text {, Veillonella } \uparrow \text {, diversity } \\
\text { of firmicutes } \downarrow \text {, firmicutes/bacteroidetes } \downarrow \text {, Clostridia } \downarrow \text {, Subdoligranulum } \downarrow\end{array}$ & {$[36]$} \\
\hline Human & $\mathrm{HCC}$ & Bacteroides $\uparrow$, Akkermansia $\downarrow$, Bifidobacterium $\downarrow$ & {$[37]$} \\
\hline Human & PLC & Altered gut microbiota & {$[38]$} \\
\hline Human & $\mathrm{HCC}$ & $\begin{array}{l}\text { Neisseria } \uparrow, \text { Enterobacteriaceae } \uparrow, \text { Veillonella } \uparrow, \text { Limnobacter } \uparrow, \text { Enterococcus } \downarrow \text {, Phyllobacterium } \downarrow \text {, Clostridium } \downarrow \text {, } \\
\text { Ruminococcus } \downarrow \text {, Coprococcus } \downarrow\end{array}$ & {$[39]$} \\
\hline Human & $\mathrm{HCC}$ & $\begin{array}{l}\text { Gut microbial a-diversity } \downarrow \text {, Proteobacteria } \uparrow \text {, Enterobacteriaceae } \uparrow, \text { Bacteroides xylanisolvens } \uparrow, \text { B. caecimuris } \uparrow \text {, } \\
\text { Ruminococcus gnavus } \uparrow, \text { Clostridium bolteae } \uparrow, \text { Veillonella parvula } \uparrow \text {, Oscillospiraceae } \downarrow \text {, Erysipelotrichaceae } \downarrow\end{array}$ & {$[40]$} \\
\hline Human & $\mathrm{HCC}$ & Klebsiella $\uparrow$, Haemophilus $\uparrow$, Alistipes $\downarrow$, Phascolarctobacterium $\downarrow$, Ruminococcus $\downarrow$ & [3] \\
\hline
\end{tabular}

So far, some experimental data obtained from human studies and animal models indicate that gut microbiota is related to HCC occurrence (Tables 1,2). Changes of gut microbiota together with their-derived products and metabolites account for the important factors to promote CLD and HCC occurrence. Here, the mechanisms of microbiome-gut-liver axis in promoting HCC occurrence are discussed as well as outlined in Figure 1.

\section{Experimental Studies}

Animal study results indicate a close association between gut microbiota and HCC (Table 1). As early as in 2010, Yu et al.[23] used the HCC toxic murine model and suggested that host microflora consumption following the treatment with antibiotic cocktail (ampicillin, vancomycin, neomycin sulfate, and metronidazole) suppressed carcinogenesis, and the HCC nodule size and number were significantly reduced relative to control mice. Consistent with the above results, Dapito et al. [24] suggested that mice that grew under the germ-free conditions had small and few HCC nodules compared with control mice. Besides, applying the low, nontoxic dose of lipopolysaccharide (LPS) for a long time significantly increased HCC size and number. In addition, they also suggested the direct involvement of toll-like receptor (TLR) 4 in the HCC pathogenic mechanism, with no significant differences in tumor morbidity $[23,24]$.

In keeping with these results, Xie et al. [25] used the mouse model of nonalcoholic steatohepatitis-HCC induced by streptozotocin-high fat diet and found the sig- nificantly altered gut microbial structure in the liver disease occurrence process. Meanwhile, diverse bacterial species, including Atopobium spp., Bacteroides acidifaciens, Bacteroides spp., Bacteroides uniformis, Bacteroides vulgatus, Clostridium xylanolyticum, Clostridium cocleatum, and Desulfovibrio spp., showed significant elevated levels within the model mice, which were in direct proportion to pathophysiological characteristics and LPS contents. As demonstrated by Zhang et al. [26], intestinal mucosal injury, intestinal inflammation, and gut dysbacteriosis were observed in the rat model of diethylnitrosamine (DEN)-induced HCC. The gut microbial imbalance is mainly manifested as the significant increases of Gramnegative bacteria Escherichia coli and Atopobium cluster including the genera Atopobium, Coriobacterium, Collinsella, Eggerthella, as well as the significant decrease of $B i$ fidobacterium, Enterococcus, and Lactobacillus species. Recently, by investigating how dietary cholesterol drives NAFLD-HCC by regulating gut microbiota as well as the corresponding metabolites, Zhang et al. [27] found that the high content of dietary cholesterol resulted in steatosis, steatohepatitis, fibrosis, and, finally, HCC in succession in the mice, and insulin resistance was also observed. The occurrence of NAFLD-HCC induced by cholesterol was related to intestinal flora dysbiosis. Typically, the microbial composition was clearly clustered depending on different stages of steatosis, steatohepatitis, or HCC. The sequential increases in Mucispirillum, Desulfovibrio, Anaerotruncus, and Desulfovibrionaceae were seen, but Bacteroides and Bifidobacterium were not seen in mice fed 


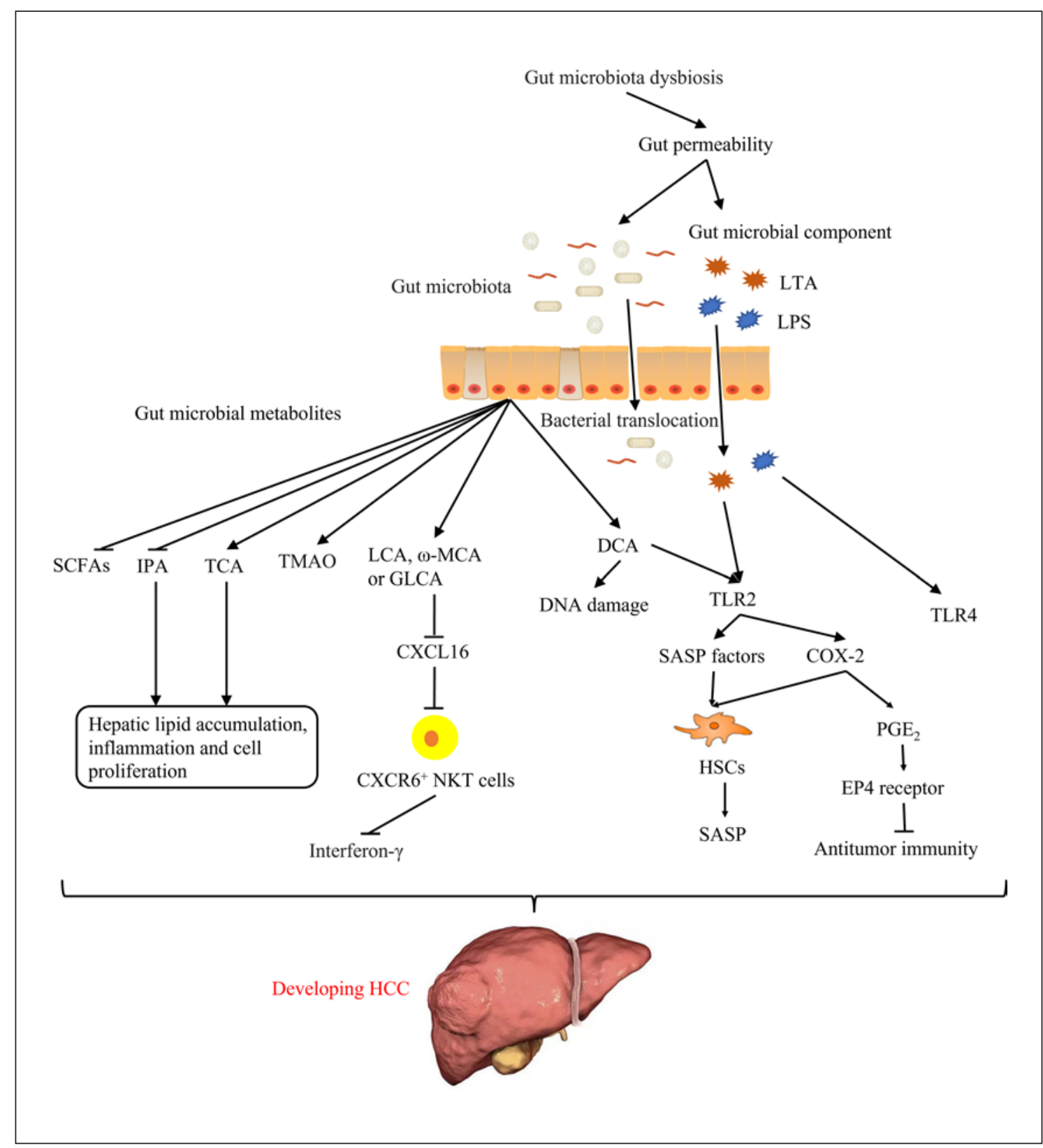

Fig. 1. The mechanism of gut microbiota on the pathogenesis of HCC. Gut microbiota dysbiosis can lead to the increase of gut permeability, which eventually can lead to microbial translocation and increasing hepatic exposure to microbiota-derived products and metabolites. Subsequently, the progression of liver disease and the development of HCC were promoted via multiple mechanisms. LPS binding with TLR4 that may be directly involved in the pathogenesis of HCC. In addition, the increase of TCA and decrease of IPA may induce hepatic lipid accumulation, inflammation and cell proliferation, which can eventually lead to HCC. Meanwhile, the increase of DCA may induce HCC development by causing hepatic DNA damage. At the same time, LTA may enhance SASP of HSCs collaboratively with DCA to upregulate the expression of SASP factors and COX-2 through TLR 2, thus creat- ing a tumor-promoting microenvironment. Furthermore, COX2-mediated $\mathrm{PGE}_{2}$ production suppresses the antitumor immunity through EP4 receptor, thereby contributing to HCC progression. Moreover, the increase of LCA, $\omega$-MCA, or GLCA may inhibit the expression of CXCL16, thereby inhibit CXCR6 ${ }^{+}$NKT cell accumulation and IFN- $\gamma$ production, which can eventually promote $\mathrm{HCC}$ development. In addition, the increase of TMAO may induce HCC development. Besides, the decrease of SCFAs may lead to HCC progression. TLR, toll-like receptor; TCA, taurocholic acid; IPA, 3-indolepropionic acid; LTA, lipoteichoic acid; SASP, senescenceassociated secretory phenotype; COX-2, cyclooxygenase-2; LCA, lithocholic acid; $\omega$-MCA, $\omega$-muricholic acid; GLCA, glycolithocholate; TMAO, trimethylamine-N-oxide; SCFAs, short-chain fatty acids; IFN, interferon. 
with the high-fat/high-cholesterol (HFHC), as confirmed in patients with hypercholesteremia. The changes in gut bacteria metabolites induced by dietary cholesterol include the reduced level of 3-indolepropionic acid and the elevated level of taurocholic acid. Germ-free mice given gavage of stools derived from high-fat/high-cholesterolfed mice had lipid accumulation, cell proliferation, and inflammation in the liver. Additionally, atorvastatin abolished the gut microbial dysbiosis induced by cholesterol while totally preventing the formation of NAFLD-HCC.

Yoshimoto et al. [28] investigated the occurrence of $\mathrm{HCC}$ in obese mice and discovered that genetic or dietary obesity caused changes in gut microbiota, thus elevating the content of secondary bile acid deoxycholic acid (DCA), the metabolite identified to induce DNA injury [29]. Meanwhile, DCA circulation in the intestine and liver enhances senescence-associated secretory phenotype (SASP) within hepatic stellate cells (HSCs) [30], while this can then produce diverse tumor-promoting and inflammatory factors in the liver, thereby enhancing HCC formation in chemical carcinogen-exposed mice. It should be noted that hindering DCA synthesis and decreasing gut bacteria can effectively prevent HCC formation in obese mice. Consistent findings are obtained in SASP inducer-deficient or senescent HSCs-depleted mice [31], which indicate the important function of DCASASP axis in HSCs during the formation of HCC induced by obesity. Similarly, Ma et al. [32] used a primary liver model as well as 3 liver metastasis models and discovered that the changes in commensal gut bacteria caused the anticancer effect in a liver-selective manner. The selectively increased hepatic $\mathrm{CXCR6}^{+}$natural killer T (NKT) cells were seen, which was not affected by the mouse strain, sex, and liver tumor present. Besides, the hepatic NKT cells accumulated exhibited the activation phenotype, which generated greater amounts of interferon- $\gamma$ when they were stimulated by antigen. According to studies in vivo that used antibody-mediated NKT-deficient and cell depletion mouse models, NKT cells inhibited the liver tumor development. Furthermore, the accumulation of NKT cells was modulated via the expression of CXCL16, the solo ligand for CXCR6, on the hepatic sinusoidal endothelial cell, which forms the lining of liver capillaries and the first barrier for the blood coming from the gut entering the liver. Primary bile acids can upregulate CXCL16, while secondary bile acids can downregulate it. The vancomycin-based antibiotic therapy can remove $\mathrm{G}^{+}$ bacteria, including those that mediate the conversion of primary bile acids to secondary bile acids, can sufficiently cause the accumulation of hepatic NKT cells, and can

The Gut Microbiome and Hepatocellular Carcinoma suppress the growth of liver tumor. Bile acid-metabolizing bacteria (Clostridium scindens) colonization and secondary bile acids (lithocholic acid or $\omega$-muricholic acid) supplementation can reverse both the accumulation of NKT cells and inhibition of liver tumor growth in mice containing the changed gut commensal bacteria. In the healthy liver tissues of primary LC cases, the level of cheno-DCA (a primary bile acid) is positively related to CXCL16 level, while glycolithocholate (a secondary bile acid) is negatively correlated with CXCL16 level, which suggests that such results may be applicable to human beings.

Recently, Loo et al. [33] reported that hepatic translocation of obesity-induced lipoteichoic acid (the $\mathrm{G}^{+}$gut microbial component) enhanced HCC formation through generating the oncogenic microenvironment. In addition, lipoteichoic acid promoted the SASP of HSCs collaboratively with DCA (the gut microbial metabolite induced by obesity) to upregulate cyclooxygenase-2 (COX2) and SASP factors via TLR-2. It was interesting that prostaglandin $\mathrm{E}_{2}\left(\mathrm{PGE}_{2}\right)$ generation induced by $\mathrm{COX}-2$ inhibits the anticancer immune response via EP4 receptor, thus facilitating HCC occurrence. Additionally, the overexpressed COX-2 and excessive $\mathrm{PGE}_{2}$ generation were observed in HSCs from HCCs patients with noncirrhotic, nonalcoholic steatohepatitis, which indicated the potentially consistent mechanism in human beings. Altogether, the COX-2 pathway induced by gut microbiota generates lipid mediators. $\mathrm{PGE}_{2}$ within the senescent HSCs in tumor microenvironment has an important function in suppressing anticancer immunity.

Taken together, these experimental studies reveal the significant alterations of gut microbiota during liver disease occurrence process. Meanwhile, these findings provided a link between the gut microbiota and their-derived products, metabolites, and immune responses in the liver. Thus, the associations of gut microbial ecology with liver pathology possibly stand for the potential diagnostic and therapeutic targets for CLD and HCC.

\section{Clinical Studies}

More and more clinical trials also show that gut microbial composition is associated with HCC occurrence. Additionally, gut microbiota has an important potential as an early biomarker to diagnose HCC. In 2016, Grąt et al. [34] discovered that gut microbial profiles related to HCC among patients with cirrhosis were featured by the elevated E. coli abundances in the feces. Meanwhile, predicting HCC presence based on fecal $E$. coli counts may be very potential. Consequently, E. coli overgrowth in the intes- 
tine possibly facilitates HCC formation. The dysbiosis degree related to primary HCC elevates as cancer development stage increases. By introducing the more comprehensive integrated index called the degree of dysbiosis $\left(D_{\text {dys }}\right), \mathrm{Ni}$ et al. [35] found that primary HCC cases had higher fecal microbial levels of pro-inflammatory bacteria compared with healthy individuals. Notably, $\mathrm{D}_{\text {dys }}$ elevated remarkably among primary HCC cases relative to normal subjects. Additionally, $\mathrm{D}_{\mathrm{dys}}$ tended to elevate as the primary HCC stage increased, even though the difference was not significant between diverse primary HCC stages. Similarly, in order to investigate gut microbial alterations at different primary LC (PLC) stages, the association of intestinal microbiota with PLC is illustrated. By comparing intestinal microbiota of PLC patients, LC patients, and healthy control subjects, Zhang et al. [36] found that the Firmicutes diversity at phylum level decreased from HC to LC and PLC patients. Across the diverse species, Enterobacter ludwigii was enriched in PLC patients, which increased by 100 folds relative to those in LC and HC groups. The Firmicutes/Bacteroidetes ratio remarkably declined as disease progressed. Meanwhile, Clostridia was the dominant gut microbial species in HC group, while Lactobacillales, Enterococcaceae, Gammaproteobacteria, and Bacilli can serve as the markers to diagnose PLC. In addition, correlation analysis was conducted to investigate the relations of gut microbial diversity with clinical factors, which showed that Veillonella was significantly positively correlated with alpha fetoprotein (AFP) of PLC patients, while Subdoligranulum was negatively correlated with AFP.

In line with these results, to explore the gut microbial characteristics related to HCC patients with NAFLD cirrhosis, by studying the gut microbial profiles, circulating mononuclear cells, inflammatory status, and intestinal permeability in HCC cases with cirrhosis induced by NAFLD, cases suffering from cirrhosis induced by NAFLD, and normal subjects were studied. Ponziani et al. [37] showed that HCC cases had elevated fecal calprotectin contents, with similar intestinal permeability to that in cases with cirrhosis without HCC. The C-C motif chemokine ligand (CCL) 3, CCL4, CCL5, interleukin 8, and interleukin 13 levels in plasma increased among HCC cases, which were related to the activation of circulating monocytes. For all patients with cirrhosis, the Streptococcus and Enterobacteriaceae were significantly enriched in fecal microbiota, while the Akkermansia abundance decreased relative to those in normal subjects. Ruminococcaceae and Bacteroides were enriched in HCC patients compared with cirrhotic patients, whereas the abundance of Bifidobacterium decreased. The abundance of Bifidobacterium and Akkermansia was negatively correlated with the calprotectin content, which in turn was related to cellular and humoral inflammatory factors. Bacteroides also came to similar results.

Liu et al. [38] demonstrated that serum trimethylamine-N-oxide (TMAO) (a choline derived metabolite produced by gut microbiota) content increased in PLC patients compared with controls. The increased trimethylamine-N-oxide content in serum predicted a higher risk of PLC. Such relation was more potent among patients showing decreased serum choline contents. More large-sample-size prospective research is warranted for confirming the above results. Likewise, to characterize gut dysbiosis in non-LC-induced HCC (NLC-HCC) and LC-induced HCC (LC-HCC) elucidates the role of gut microbiota in HCC pathogenic mechanism. By comparing fecal microbiota in hepatitis patients, LC patients, LCHCC patients, NLC-HCC patients, and healthy controls, Zheng et al. [39] discovered the remarkable reduction of fecal microbial $\alpha$-diversity among LC cases; besides, there were significant differences in 27 genera and 3 phyla between LC and other groups (hepatitis, HCC, and normal groups). Besides, beta-diversity was largely different in LC compared with other groups. HCC group had markedly higher gut microbial diversity compared with LC group. Characterizing the fecal microbiota of LC-HCC and NLC-HCC, the fecal microbial diversity of LC-HCC group, but not NLC-HCC group, significantly increased compared with LC group. There were 13 genera related to HCC tumor size. The abundances of butyrate-producing genera (Clostridium, Ruminococcus, and Coprococcus) declined, whereas those of LPS-producing genera (Neisseria, Enterobacteriaceae, and Veillonella) increased among LC-HCC patients. Besides, 3 biomarkers (Enterococcus, Phyllobacterium, Limnobacter) might be utilized to diagnose disease, accurately.

In recent, by characterizing gut microbiota among cases suffering from NAFLD-induced cirrhosis, in the presence or absence of HCC, and evaluating the role in peripheral immune reaction based on the in vitro model, Behary et al. [40] found that dysbiosis characterized microbial communities in cases suffering from NAFLD-induced cirrhosis, and the compositions and functions were changed in the process of HCC development. For NAFLDHCC cases, the microbial gene functions promote the generation of short-chain fatty acids (SCFAs), as verified through metabolomic study. Moreover, studies in vitro indicate that the NAFLD-HCC microbiota-derived, rather than control group-derived bacterial extracts, trigger 
Table 3. New potential gut microbial biomarkers for the early diagnosis of HCC

\begin{tabular}{llll}
\hline Models & Disease & Gut microbial biomarkers & Reference \\
\hline Human & HCC & Escherichia coli & {$[34]$} \\
Human & HCC & Enterococcaceae, Lactobacillales, Bacilli and Gammaproteobacteria & {$[36]$} \\
Human & HCC & Enterococcus, Limnobacter, and Phyllobacterium & {$[39]$} \\
Human & HCC & 30 microbial markers & {$[3]$} \\
\hline
\end{tabular}

30 microbial markers: OTU10, OTU12, OTU28, OTU57, OTU58, OTU63, OTU78, OTU86, OTU87, OTU96, OTU97, OTU128, OTU136, OTU209, OTU285, OTU291, OTU310, OTU372, OTU373, OTU427, OTU451, OTU624, OTU664, OTU748, OTU927, OTU968, OTU976, OTU1032, OTU1091, and OTU1294.

the T-cell immunosuppressive phenotype, which is characterized by the attenuated $\mathrm{CD} 8^{+} \mathrm{T}$ cells and expanded regulatory T cells.

All in all, as suggested by these clinical studies, gut microbiota has a key function in HCC occurrence and development. Furthermore, gut microbiota may be early diagnostic biomarker of HCC, and its manipulation is an efficient strategy to prevent and treat HCC.

\section{Gut Microbiota for the Early Diagnosis of HCC}

Early diagnosis of HCC is accompanied by a variety of treatment options and typically leads to good outcomes. Biomarkers such as AFP, des-gamma-carboxy prothrombin, and Lens culinaris agglutinin A-reactive fraction of AFP (AFP-L3) are identified to be the markers specific to HCC $[41,42]$. The novel potential biomarkers, including aldo-keto reductase family 1 member 10 [43], have been studied to diagnose HCC and predict its prognosis. Notably, gut microbiota as a novel biomarker is reported. Gut microbiota is advantageous in diagnosing disease, like the noninvasiveness, high efficiency, and accuracy. Gut microbial alterations may serve as biomarkers of HCC disease since they are related to liver disease development, ranging from cirrhosis/fibrosis to cancer [44, 45]. In recent, some studies regarding the association of gut microbiota with HCC have preliminarily indicated that it is important to identify microbiome biomarkers based on gut microbial alterations in CLD to diagnose HCC at an early stage (Table 3 ).

Recently, in order to characterize gut microbiome among HCC cases and assess the possibility to use it as the noninvasive biomarker to diagnose HCC, by characterizing gut microbiota, identifying the biomarkers, and constructing the HCC classifiers among early HCC patients, cirrhosis patients, and normal subjects, validating the findings in normal subjects, early HCC cases, and advanced HCC cases, as well as further verifying diagnosis potential in HCC from Xinjiang and Zhengzhou, Ren et al. [3] found that fecal microbial diversity in early HCC with cirrhosis showed an increase compared with that in cirrhosis. In addition, compared with cirrhosis, the abundance of phylum Actinobacteria elevated in early HCC. Accordingly, the abundances of 13 genera such as Parabacteroides and Gemmiger increased in early HCC compared with cirrhosis. By contrast, the abundances of $\mathrm{Bu}-$ tyrate-producing genera showed a decrease, whereas those of LPS-producing genera increased in early HCC patients compared with normal subjects. Moreover, the authors identified the optimal 30 microbial markers between non-HCC and early HCC cases. Notably, the potent potential of gut microbial markers in diagnosing early or even advanced HCC was verified. Importantly, microbial markers successfully achieved a cross-region verification of HCC in Northwest China and Central China.

Altogether, the study has first characterized gut microbiome among HCC cases established the diagnosis model, and validated the use of microbial markers to successfully diagnose HCC in a cross-region manner. Gut microbiota-targeted biomarkers are the candidate noninvasive approaches to diagnose HCC in the early stage.

\section{Targeting Microbiota for HCC Prevention}

At present, no available treatment can be applied to prevent HCC in addition to the treatment of underlying disease. As the gut-microbiota-liver axis contributes greatly to CLD and HCC development, it is a promising target in prevention measures (Fig. 2), supported by many preclinical articles showing that the HCC incidence dramatically decreases by about $80 \%$ in rat and 


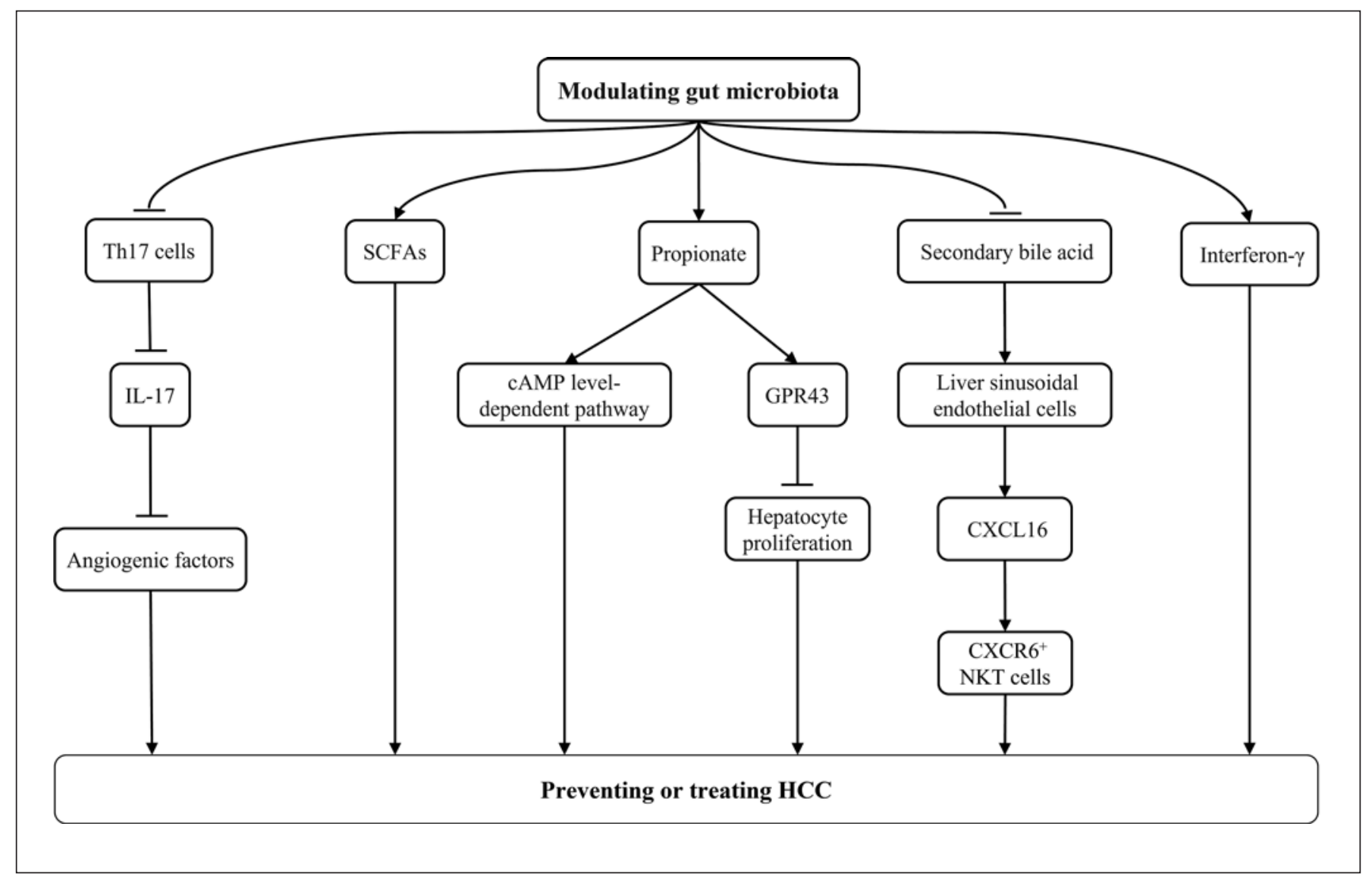

Fig. 2. The mechanism of gut microbiota target therapy to HCC. Modulating gut microbiota may suppress Th17 cell differentiation and their secreted IL-17 weakened the angiogenesis, and subsequently suppressed HCC progression. In addition, regulating gut microbiota may promote SCFAs production and ultimately can inhibit HCC development. Meanwhile, modulating gut microbiota may increase the production of propionate that may improve HCC not only through a cAMP level-dependent pathway but also

mouse models $[23,24,28]$. Further, several small-scale clinical trials also reveal that antibiotic treatment like rifaximin and norfloxacin can extend the survival time of cirrhotic cases [46-49]. Targeting the gut microbiota for HCC prevention is particularly attractive, since it can use some approaches with a high safety profile and low risk of severe adverse effects, like probiotics, prebiotics, synbiotics, fecal microbiota transplantation (FMT), and antibiotics.

\section{Probiotics}

Probiotics are live microorganisms, which benefit the host if they are given in sufficient doses [50]; for instance, they improve gut microbial balance and stimulate bacte- by binding with GPR43. Furthermore, regulating gut microbiota may induce an anti-HCC effect, with an increase of hepatic $\mathrm{CXCR}^{+}{ }^{\mathrm{NKT}}$ cells and heightened IFN- $\gamma$ production. At the same time, CXCR6 ${ }^{+}$NKT cell accumulation was regulated by CXCL16 expression of liver sinusoidal endothelial cells, which was controlled by gut microbiome-mediated primary-to-secondary bile acid conversion. cAMP, cyclic Adenosine monophosphate; GPR, G protein coupled receptor; IFN, interferon; IL, interleukin. rial products and metabolites [51]. The popularity of probiotics has expanded exponentially recently, due to their health promoting effects and their abilities to prevent or treat different diseases [52-61]. Likewise, many studies suggest that probiotics are effective on the treatment of liver diseases in animal models and human beings. At present, probiotics are just studied based on the murine HCC models, and relevant data in human beings are unavailable (Table 4).

As early as 2012, Zhang et al. [26] found that VSL\#3 (containing Bifidobacterium breve, Streptococcus thermophiles, Bifidobacterium infantis, Bifidobacterium longum, Lactobacillus plantarum, Lactobacillus acidophilus, Lactobacillus delbrueckii subsp. bulgaricus, Lactobacillus pa- 
Table 4. Targeting microbiota therapeutic strategies for HCC

\begin{tabular}{|c|c|c|c|c|}
\hline Mice & DEN induced HCC & VSL\#3 & $\begin{array}{l}\mathrm{G}^{-} \text {bacteria groups } \downarrow \text {, Escherichia coli } \downarrow \text {, Atopobium cluster } \\
\downarrow, \text { Bacteroides fragilis group } \downarrow \text {, Prevotella } \downarrow\end{array}$ & {$[26]$} \\
\hline Mice & Hepa1-6 transplanted tumor & Prohep & $\begin{array}{l}\text { Alistipes } \uparrow, \text { Butyricimonas } \uparrow, \text { Mucispirillum } \uparrow, \text { Oscillibacter } \uparrow, \\
\text { Parabacteroides } \uparrow, \text { Paraprevotella } \uparrow, \text { Prevotella } \uparrow, \\
\text { Bacteroides fragilis } \uparrow \text {, Alistipes shahii } \uparrow, \text { Parabacteroides } \\
\text { distasonis } \uparrow, \text { Akkermansia muciniphila } \uparrow\end{array}$ & {$[62]$} \\
\hline Mice & DEN induced HCC & $\begin{array}{l}\text { An antibiotic cocktail (ampicillin, } \\
\text { vancomycin, neomycin sulfate, } \\
\text { and metronidazole) }\end{array}$ & No & {$[23]$} \\
\hline Mice & DEN-CCL4 induced HCC & $\begin{array}{l}\text { An antibiotic cocktail (ampicillin, } \\
\text { neomycin, metronidazole and } \\
\text { vancomycin) }\end{array}$ & No & {$[24]$} \\
\hline Mice & $\begin{array}{l}\text { MYC transgenic spontaneous } \\
\text { HCC }\end{array}$ & $\begin{array}{l}\text { An antibiotic cocktail (vancomycin, } \\
\text { neomycin, and primaxin) or } \\
\text { vancomycin }\end{array}$ & Verrucomicrobiales $\uparrow$, Clostridiales $\downarrow$, Bacteroidales $\downarrow$ & {$[32]$} \\
\hline Mice & Inulin-induced HCC & Vancomycin & $\begin{array}{l}\text { Lachnospiraceae } \downarrow \text {, Ruminococcaceae } \downarrow \text {, Bifidobacteria } \downarrow \text {, } \\
\text { Clostridium cluster XIVa } \downarrow\end{array}$ & {$[68]$} \\
\hline
\end{tabular}

VSL\#3: Streptococcus thermophiles, Bifidobacterium breve, B. longum, B. infantis, Lactobacillus acidophilus, L. plantarum, L. paracasei, and L. delbrueckil subsp. Bulgaricus. Prohep: Lactobacillus rhamnosusGG, Escherichia coli Nissle 1917 and heat-inactivated VSL\#3. G', gram-negative.

racasei) administration alleviated HCC occurrence induced by DEN through the restoration of gut homeostasis and the mitigation of hepatic and intestinal inflammation, thereby preventing cirrhosis from progressing into HCC.

Recently, Li et al. [62] discovered the effect of probiotics on inhibiting HCC development in mouse model. Administration of probiotics mixture Prohep (comprising heat-inactivated VSL\#3, E. coli Nissle 1917, and Lactobacillus rhamnosus GG) into mice bearing tumor xenografts reduced liver tumor size, and shifted gut microbial composition to beneficial bacteria including Oscillibacter and Prevotella, as well as anti-inflammatory factors produced by the above bacteria. Apart from the reduced tumor size, administration of probiotics can decrease the production of angiogenic factors. With regard to the underlying pathways involved in the mechanism, mice administered with probiotics had decreased gut Th17 cell level and Th17 recruitment into the tumor site. Moreover, probiotics' antitumor activity was related to the generation of SCFAs, as evidenced by the enriched SCFA-related pathway discovered in mice treated with probiotics. Altogeth- er, probiotics treatment can regulate the microbiota and affect the regulation of the $\mathrm{T}$-cell differentiation in the gut, while these then change the pro-inflammatory cytokine contents within the extraintestinal tumor microenvironment.

\section{Prebiotics}

Prebiotics represent the nonabsorbent oligosaccharide substances, including lactulose. They function to accelerate beneficial bacterial growth and suppress harmful bacterial growth, thus adjusting the balance of gut microbiota [63]; in addition, they can lead to the production of SCFAs and regulate the immune response. Thus, prebiotics may prevent or treat HCC. But so far, there is only one study that has investigated the effects of prebiotics on HCC (Table 4). Bindels et al. [64] demonstrated that treatment with inulin-type fructans decreased the infiltration of hepatic BaF3 cells, mitigated inflammation while increasing portal propionate content in mice given transplantation of Bcr-Abl-transfected BaF3 cells. Besides, propionate can suppress the growth of $\mathrm{BaF} 3$ cells in vitro by the cAMP-dependent pathway. Further, when 
free fatty acid receptor 2 , the Gi/Gq-protein-coupled receptor also referred to as GPR43 that binds to propionate, is activated, it can decrease the proliferation of $\mathrm{BaF} 3$ as well as other human cancer cells. All in all, these results support prebiotics as the novel antitumor treatment strategy.

\section{Synbiotics}

Synbiotics are the complex constituted by prebiotics and probiotics, which have been suggested to show higher efficient than prebiotics or probiotics alone [65]. Similarly, synbiotics may improve HCC. At present, no study has investigated the ameliorating effect of synbiotics on HCC.

\section{Fecal Microbiota Transplantation}

FMT is a process that administers fecal matter solution obtained from a normal donor to the recipient intestinal tract, so as to directly change the microbial composition of the latter and induce the health benefits or treat a certain disorder $[53,54]$. Since FMT has successfully been utilized among cases suffering from $C$. difficile infection, which can restore eubiosis and achieve better clinical improvements than those of standard antibiotic therapy, FMT is currently being assessed in some clinical studies to treat numerous other disorders, such as irritable bowel syndrome, inflammatory bowel disease, cardiovascular disease, and metabolic diseases $[53,54,58,66]$. Recently, FMT has been shown to have a role in some types of cancer, such as non-small cell lung cancer, colorectal cancer, and melanoma [59, 67]. In particular, FMT can enhance efficacy of immune checkpoint inhibitors therapy against these types of cancer. Likewise, FMT may treat or prevent HCC. Furthermore, FMT to enhance the antitumor effect of immune checkpoint inhibitors is a potential strategy for HCC treatment. However, there is currently no data to support this premise, and a number of hurdles must be overcome.

\section{Antibiotics}

Since antibiotics can target several pathways through which the gut microbiota promotes HCC occurrence and development (Table 4), they may be one of the most effective strategies to interrupt the tumor-promoting gutliver axis in CLD. Reducing the total gut bacterial quantity and removing the bacteria with a highly translocated ability can decrease bacterial translocation, thus inhibiting pro-inflammatory signals arising from leaky gut. Meanwhile, selective antibiotics can suppress generation of bacterial metabolites that promote HCC, like DCA, through decreasing the gut bacterial quantity related to the production of these metabolites [28].

Continuous intestinal sterilization through the oral antibiotic cocktail including neomycin, ampicillin, vancomycin, and metronidazole efficiently decreased the number and size of HCC tumors induced by DMBAHFD or DEN-CCL4 in mice $[24,28]$. Consistent with these observations, $\mathrm{Ma}$ et al. [32] found that the antibiotic cocktail (ABX, containing vancomycin, primaxin, neomycin) or vancomycin treatment elicited the liver-selective anticancer effect, with an increase of hepatic CXCR6 $^{+}$NKT cells and enhanced interferon- $\gamma$ synthesis upon antigen stimulation in MYC transgenic spontaneous HCC mouse model. Meanwhile, NKT cells mediated liver-selective tumor inhibition. The accumulation of NKT cells was regulated by CXCL16 expression of liver sinusoidal endothelial cells, which was controlled through intestinal microbiome-mediated the conversion of primary bile acids to secondary bile acids.

Recently, Singh et al. [68] demonstrated that vancomycin treatment could suppress the development of LC in inulin-fed mice with TLR5 (T5KO) deficiency. Meanwhile, vancomycin treatment led to the selective depletion of gut microbiota, including Bifidobacteria of phylum Actinobacteria, as well as $\mathrm{G}^{+}$Lachnospiraceae and Ruminococcaceae of phylum Firmicutes, which ferment fibers, as well as Clostridium cluster XIVa, which generate the secondary bile acids. The lack of LC in vancomycintreated mice is closely related to the large loss circulating secondary bile acids.

\section{Conclusions and Future Perspective}

The microbiota-gut-liver axis has a key function in liver disease pathogenic mechanism, like HCC. Increasing evidence has supported the role of the gut microbiota in HCC occurrence and development. Meanwhile, gut microbiota is the potential biomarker used to diagnose HCC early. Therefore, manipulating gut microbiota may be a new approach for HCC treatment or prevention. Probiotics, prebiotics, synbiotics, FMT, and antibiotics may be the innovative, cost-effective, safe, and noninvasive manners for HCC prevention and treatment. Nonetheless, there are numerous problems to be solved with regard to microbiota's effect on HCC genesis. Further research is warranted to investigate on how the microbiota are assembled as well as on which factors contribute to their long-run stability in both health and illness. Meanwhile, more efforts are needed to develop treat- 
ment targeting specific gut microbial species or gut microbiota-derived metabolites. Although high-throughput sequencing can be conducted to detect gut microbiota existing within the sample with no need of culture, its results just represent the association between gut microbiota and disease. It is still a significant challenge to move from association to causation. Some specific gut microbial species should be cultured for causation test. As a result, culturomic technique is greatly needed. Furthermore, due to the complicated gut microbial communities, multi-omics analysis that includes transcriptomics, metabolomics, and proteomics allows to overview the whole disease landscape. Additionally, many clinical trials must concentrate on identifying the best bacterial biomarkers to diagnose HCC in an early stage. Parallelly, more well-designed clinical trials evaluating gut microbiota-mediated therapies are necessary for guaranteeing the safe outcome and improve outcomes of HCC treatment. At last, in order to gain the recognition of the wider medical community and investigate the possibility of using probiotics as an alternative therapeutic method for cancer, more laboratory-based mechanistic studies and extensive human clinical trials are needed to evaluate the intestinal microbiota and appropriately selected useful bacterial strains. Therefore, more efforts are needed to translate the current knowledge regarding the function of microbiota-gut-liver axis in promoting HCC into diagnostic, prognostic, and therapeutic strategies in patients.

\section{Conflict of Interest Statement}

The authors report no conflicts of interest.

\section{Funding Sources}

This work was supported by Science Research Start-up Fund for Doctor of Shanxi Medical University (Grant No. XD1807), Science Research Start-up Fund for Doctor of Shanxi Province (Grant No. SD1807), the Scientific and Technological Innovation Programs of Higher Education Institutions in Shanxi (Grant No. 2019L0425), the Shanxi Province Science Foundation for Youths (Grant No. 201901D211314), the Natural Science Foundation of China (Grant No. 82160159), Endocrine Clinical Medical Center of Yunnan Province (Grant No. ZX2019-02-02), and Fund of Medical Leader in Yunnan Province (Grant No. L-201609).

\section{Author Contributions}

Yongbo Kang conceived, collected data, drafted, and approved the final manuscript. Yue Cai and Ying Yang reviewed, provided critical comments, and approved the final manuscript.

\section{References}

1 Bray F, Ferlay J, Soerjomataram I, Siegel RL, Torre LA, Jemal A. Global cancer statistics 2018: GLOBOCAN estimates of incidence and mortality worldwide for 36 cancers in 185 countries. CA Cancer J Clin. 2018;68(6):394424.

2 Rao BC, Lou JM, Wang WJ, Li A, Cui GY, Yu ZJ, et al. Human microbiome is a diagnostic biomarker in hepatocellular carcinoma. Hepatobiliary Pancreat Dis Int. 2020;19(2):10915.

3 Ren Z, Li A, Jiang J, Zhou L, Yu Z, Lu H, et al. Gut microbiome analysis as a tool towards targeted non-invasive biomarkers for early hepatocellular carcinoma. Gut. 2019;68(6): 1014-23.

4 GBD 2017 DALYs and HALE Collaborators. Global, regional, and national disability-adjusted life-years (DALYs) for 359 diseases and injuries and healthy life expectancy (HALE) for 195 countries and territories, 1990-2017: a systematic analysis for the Global Burden of Disease Study 2017. Lancet. 2018;392(10159): 1859-922.
5 Balogh J, Victor D 3rd, Asham EH, Burroughs SG, Boktour M, Saharia A, et al. Hepatocellular carcinoma: a review. J Hepatocell Carcinoma. 2016;3:41-53.

6 Mokdad AA, Hester CA, Singal AG, Yopp AC. Management of hepatocellular in the United States. Chin Clin Oncol. 2017;6(2):21.

7 Yu LX, Schwabe RF. The gut microbiome and liver cancer: mechanisms and clinical translation. Nat Rev Gastroenterol Hepatol. 2017; 14(9):527-39.

8 Sia D, Villanueva A, Friedman SL, Llovet JM. Liver cancer cell of origin, molecular class, and effects on patient prognosis. Gastroenterology. 2017;152(4):745-61.

9 Zhang C, Yang M, Ericsson AC. Antimicrobial peptides: potential application in liver cancer. Front Microbiol. 2019;10:1257.

10 Rooks MG, Garrett WS. Gut microbiota, metabolites and host immunity. Nat Rev Immunol. 2016;16(6):341-52.

11 Tremaroli V, Bäckhed F. Functional interactions between the gut microbiota and host metabolism. Nature. 2012;489(7415):242-9.

12 Schroeder BO, Bäckhed F. Signals from the gut microbiota to distant organs in physiology and disease. Nat Med. 2016;22(10):1079-89.
13 Erstad DJ, Tanabe KK. Hepatocellular carcinoma: early-stage management challenges. J Hepatocell Carcinoma. 2017;4:81-92.

$14 \mathrm{Yu}$ J, Feng Q, Wong SH, Zhang D, Liang QY, Qin Y, et al. Metagenomic analysis of faecal microbiome as a tool towards targeted noninvasive biomarkers for colorectal cancer. Gut. 2017;66(1):70-8.

15 Qin J, Li Y, Cai Z, Li S, Zhu J, Zhang F, et al. A metagenome-wide association study of gut microbiota in type 2 diabetes. Nature. 2012; 490(7418):55-60.

16 Qin N, Yang F, Li A, Prifti E, Chen Y, Shao L, et al. Alterations of the human gut microbiome in liver cirrhosis. Nature. 2014;513(7516): 59-64.

17 Ren Z, Jiang J, Xie H, Li A, Lu H, Xu S, et al Gut microbial profile analysis by MiSeq sequencing of pancreatic carcinoma patients in China. Oncotarget. 2017;8(56):95176-91.

18 Pasini E, Aquilani R, Testa C, Baiardi P, Angioletti S, Boschi F, et al. Pathogenic gut flora in patients with chronic heart failure. JACC Heart Fail. 2016;4(3):220-7. 
19 Jacqueline C, Brazier L, Faugère D, Renaud F, Thomas F, Roche B. Can intestinal microbiota be associated with non-intestinal cancers? Sci Rep. 2017;7(1):12722.

20 Cabrera R, Nelson DR. Review article: the management of hepatocellular carcinoma. Aliment Pharmacol Ther. 2010;31(4):461-76.

21 El-Serag HB. Hepatocellular carcinoma. N Engl J Med. 2011;365(12):1118-27.

22 Mittal S, El-Serag HB. Epidemiology of hepatocellular carcinoma: consider the population. J Clin Gastroenterol. 2013;47 Suppl(0): S2-6.

23 Yu LX, Yan HX, Liu Q, Yang W, Wu HP, Dong W, et al. Endotoxin accumulation prevents carcinogen-induced apoptosis and promotes liver tumorigenesis in rodents. Hepatology. 2010;52(4):1322-33.

24 Dapito DH, Mencin A, Gwak GY, Pradere JP, Jang MK, Mederacke I, et al. Promotion of hepatocellular carcinoma by the intestinal microbiota and TLR4. Cancer Cell. 2012;21(4): $504-16$.

25 Xie G, Wang X, Liu P, Wei R, Chen W, Rajani $\mathrm{C}$, et al. Distinctly altered gut microbiota in the progression of liver disease. Oncotarget. 2016;7(15):19355-66.

26 Zhang HL, Yu LX, Yang W, Tang L, Lin Y, Wu $\mathrm{H}$, et al. Profound impact of gut homeostasis on chemically-induced pro-tumorigenic inflammation and hepatocarcinogenesis in rats. J Hepatol. 2012;57(4):803-12.

27 Zhang X, Coker OO, Chu ES, Fu K, Lau HCH, Wang YX, et al. Dietary cholesterol drives fatty liver-associated liver cancer by modulating gut microbiota and metabolites. Gut. 2021; 70(4):761-74.

28 Yoshimoto S, Loo TM, Atarashi K, Kanda H, Sato S, Oyadomari S, et al. Obesity-induced gut microbial metabolite promotes liver cancer through senescence secretome. Nature. 2013;499(7456):97-101.

29 Ridlon JM, Kang DJ, Hylemon PB. Bile salt biotransformations by human intestinal bacteria. J Lipid Res. 2006;47(2):241-59.

30 Friedman SL. Hepatic stellate cells: protean, multifunctional, and enigmatic cells of the liver. Physiol Rev. 2008;88(1):125-72.

31 Orjalo AV, Bhaumik D, Gengler BK, Scott GK, Campisi J. Cell surface-bound IL-1alpha is an upstream regulator of the senescence-associated IL-6/IL-8 cytokine network. Proc Natl Acad Sci U S A. 2009; 106(40):17031-6.

32 Ma C, Han M, Heinrich B, Fu Q, Zhang Q, Sandhu M, et al. Gut microbiome-mediated bile acid metabolism regulates liver cancer via NKT cells. Science. 2018;360(6391): eaan5931.

33 Loo TM, Kamachi F, Watanabe Y, Yoshimoto S, Kanda H, Arai Y, et al. Gut microbiota promotes obesity-associated liver cancer through PGE(2)-mediated suppression of antitumor immunity. Cancer Discov. 2017; 7(5):522-38.
34 Grąt M, Wronka KM, Krasnodębski M, Masior $€$, Lewandowski Z, Kosińska I, et al. Profile of gut microbiota associated with the presence of hepatocellular cancer in patients with liver cirrhosis. Transplant Proc. 2016;48(5): 1687-91.

35 Ni J, Huang R, Zhou H, Xu X, Li Y, Cao P, et al. Analysis of the relationship between the degree of dysbiosis in gut microbiota and prognosis at different stages of primary hepatocellular carcinoma. Front Microbiol. 2019; 10:1458.

36 Zhang L, Wu YN, Chen T, Ren CH, Li X, Liu GX. Relationship between intestinal microbial dysbiosis and primary liver cancer. Hepatobiliary Pancreat Dis Int. 2019;18(2):149-57.

37 Ponziani FR, Bhoori S, Castelli C, Putignani L, Rivoltini L, Del Chierico F, et al. Hepatocellular carcinoma is associated with gut microbiota profile and inflammation in nonalcoholic fatty liver disease. Hepatology. 2019; 69(1):107-20

38 Liu ZY, Tan XY, Li QJ, Liao GC, Fang AP, Zhang DM, et al. Trimethylamine N-oxide, a gut microbiota-dependent metabolite of choline, is positively associated with the risk of primary liver cancer: a case-control study. Nutr Metab. 2018;15:81.

39 Zheng R, Wang G, Pang Z, Ran N, Gu Y, Guan $\mathrm{X}$, et al. Liver cirrhosis contributes to the disorder of gut microbiota in patients with hepatocellular carcinoma. Cancer Med. 2020; 9(12):4232-50.

40 Behary J, Amorim N, Jiang XT, Raposo A, Gong L, McGovern E, et al. Gut microbiota impact on the peripheral immune response in non-alcoholic fatty liver disease related hepatocellular carcinoma. Nat Commun. 2021; 12(1):187

41 Toyoda H, Kumada T, Tada T, Sone Y, Kaneoka Y, Maeda A. Tumor markers for hepatocellular carcinoma: simple and significant predictors of outcome in patients with HCC. Liver Cancer. 2015;4(2):126-36.

42 Zhao YJ, Ju Q, Li GC. Tumor markers for hepatocellular carcinoma. Mol Clin Oncol. 2013;1(4):593-8.

43 DiStefano JK, Davis B. Diagnostic and prognostic potential of AKR1B10 in human hepatocellular carcinoma. Cancers. 2019;11(4): 486.

44 Meng X, Li S, Li Y, Gan RY, Li HB. Gut microbiota's relationship with liver disease and role in hepatoprotection by dietary natural products and probiotics. Nutrients. 2018; 10(10): 1457.

45 Ponziani FR, Nicoletti A, Gasbarrini A, Pompili M. Diagnostic and therapeutic potential of the gut microbiota in patients with early hepatocellular carcinoma. Ther Adv Med Oncol. 2019;11:1758835919848184.

46 Fernández J, Navasa M, Planas R, Montoliu S, Monfort D, Soriano G, et al. Primary prophylaxis of spontaneous bacterial peritonitis delays hepatorenal syndrome and improves survival in cirrhosis. Gastroenterology. 2007; 133(3):818-24
47 Vlachogiannakos J, Viazis N, Vasianopoulou P, Vafiadis I, Karamanolis DG, Ladas SD. Long-term administration of rifaximin improves the prognosis of patients with decompensated alcoholic cirrhosis. J Gastroenterol Hepatol. 2013;28(3):450-5.

48 Elfert A, Abo Ali L, Soliman S, Ibrahim S, Abd-Elsalam S. Randomized-controlled trial of rifaximin versus norfloxacin for secondary prophylaxis of spontaneous bacterial peritonitis. Eur J Gastroenterol Hepatol. 2016; 28(12):1450-4.

49 Sharma BC, Sharma P, Lunia MK, Srivastava S, Goyal R, Sarin SK. A randomized, doubleblind, controlled trial comparing rifaximin plus lactulose with lactulose alone in treatment of overt hepatic encephalopathy. Am J Gastroenterol. 2013;108(9):1458-63.

50 Culligan EP, Hill C, Sleator RD. Probiotics and gastrointestinal disease: successes, problems and future prospects. Gut Pathog. 2009; $1(1): 19$.

51 Bengmark S. Bioecologic control of the gastrointestinal tract: the role of flora and supplemented probiotics and synbiotics. Gastroenterol Clin North Am. 2005;34(3):413-36, viii.

52 Sanders ME, Guarner F, Guerrant R, Holt PR, Quigley EM, Sartor RB, et al. An update on the use and investigation of probiotics in health and disease. Gut. 2013;62(5):787-96.

53 Kang Y, Cai Y. Gut microbiota and obesity: implications for fecal microbiota transplantation therapy. Hormones. 2017;16(3):22334.

54 Kang Y, Cai Y. Gut microbiota and hepatitis-B-virus-induced chronic liver disease: implications for faecal microbiota transplantation therapy. J Hosp Infect. 2017; 96(4):342-8.

55 Kang Y, Cai Y. Altered gut microbiota in HIV infection: future perspective of fecal microbiota transplantation therapy. AIDS Res Hum Retroviruses. 2019;35(3):229-35.

56 Kang Y, Cai Y. Gut microbiota and hypertension: from pathogenesis to new therapeutic strategies. Clin Res Hepatol Gastroenterol. 2018;42(2):110-7.

57 Kang Y, Cai Y, Pan W. Change in gut microbiota for eczema: implications for novel therapeutic strategies. Allergol Immunopathol. 2018;46(3):281-90.

58 Kang Y, Kang X, Zhang H, Liu Q, Yang H, Fan W. Gut microbiota and Parkinson's disease: implications for faecal microbiota transplantation therapy. ASN Neuro. 2021 Jan-Dec;13: 17590914211016217.

59 Kang Y, Pan W, Cai Y. Gut microbiota and colorectal cancer: insights into pathogenesis for novel therapeutic strategies. Z Gastroenterol. 2017;55(9):872-80.

60 Kang YB, Cai Y, Zhang H. Gut microbiota and allergy/asthma: from pathogenesis to new therapeutic strategies. Allergol Immunopathol. 2017;45(3):305-9. 
61 Leblanc M. The administration of probiotics and fermented products containing lactic acid bacteria exert beneficial effects against intestinal and non-intestinal cancers. J Food Nutr Disor. 2014. Epub ahead of print.

62 Li J, Sung CY, Lee N, Ni Y, Pihlajamäki J, Panagiotou $\mathrm{G}$, et al. Probiotics modulated gut microbiota suppresses hepatocellular carcinoma growth in mice. Proc Natl Acad Sci USA. 2016;113(9):E1306-15.
63 Fotiadis CI, Stoidis CN, Spyropoulos BG, Zografos ED. Role of probiotics, prebiotics and synbiotics in chemoprevention for colorectal cancer. World J Gastroenterol. 2008;14(42): 6453-7.

64 Bindels LB, Porporato P, Dewulf EM, Verrax J, Neyrinck AM, Martin JC, et al. Gut microbiota-derived propionate reduces cancer cell proliferation in the liver. Br J Cancer. 2012; 107(8):1337-44.

65 Roberfroid M, Gibson GR, Hoyles L, McCartney AL, Rastall R, Rowland I, et al. Prebiotic effects: metabolic and health benefits. $\mathrm{Br} J$ Nutr. 2010;104(Suppl 2):S1-63.
66 Smits LP, Bouter KE, de Vos WM, Borody TJ, Nieuwdorp M. Therapeutic potential of fecal microbiota transplantation. Gastroenterology. 2013;145(5):946-53.

67 Kang YB, Cai Y. Faecal microbiota transplantation enhances efficacy of immune checkpoint inhibitors therapy against cancer. World J Gastroenterol. 2021;27(32):5362-75.

68 Singh V, Yeoh BS, Abokor AA, Golonka RM, Tian Y, Patterson AD, et al. Vancomycin prevents fermentable fiber-induced liver cancer in mice with dysbiotic gut microbiota. Gut Microbes. 2020;11(4):1077-91. 